\title{
NOTE ON THE PARAMETRIC REPRESENTATION OF AN ARBITRARY CONTINUOUS CURVE.
}

\author{
BY PROFESSOR DUNHAM JACKSON.
}

(Read before the American Mathematical Society, April 28, 1917.)

A continuous curve may be defined by a pair of equations

$$
x=f(t), \quad y=\varphi(t),
$$

where the functions $f$ and $\varphi$ are defined and continuous throughout some interval, say for $0 \leqq t \leqq 1$, and are not both constant throughout that interval. It is sometimes an aid to the imagination to suppose that they are not simultaneously constant throughout any subinterval of their interval of definition. The question naturally arises, whether this implies a restriction on the curve itself, or merely on the particular parametric representation employed. It is obvious that if there are only a finite number of intervals where $f$ and $\varphi$ are constant together, a suitable change of parameter will eliminate such intervals entirely. The present note offers a proof that this is true in all cases. A decidedly different proof has been given by Fréchet;* it is believed that the method of treatment here adopted is considerably more elementary than his, as far as obtaining the indicated result is concerned, though it does not yield certain additional knowledge which is incidental to his presentation.

On careful scrutiny, it appears that the problem of a satisfactory definition of the identity of two curves is not an altogether simple one; $\dagger$ to avoid a discussion of this point, the result is stated in the following form, which is free of ambiguity:

THEOREM. Let a pair of functions

$$
x=f(t), \quad y=\varphi(t)
$$

be defined and continuous for $0 \leqq t \leqq 1$. Then it is possible to

* Fréchet, "Sur quelques points du calcul fonctionnel," Rendiconti del Circolo Matematico di Palermo, vol. 22 (1906), pp. 1-74; pp. 58-59, 67-70.

† Cf. Fréchet, loc. cit., pp. 51-53. 
define a function $\sigma(t)$, single-valued, continuous, and nondecreasing for $0 \leqq t \leqq 1$, and a pair of functions $F(\tau), \Phi(\tau)$, single-valued and continuous for $\sigma(0) \leqq \tau \leqq \sigma(1)$, so that the two latter functions are not simultaneously constant throughout any interval $\tau_{1} \leqq \tau \leqq \tau_{2}$, where $\tau_{1}<\tau_{2}$, and so that

$$
F(\sigma)=f(t), \quad \Phi(\sigma)=\varphi(t),
$$

identically for $0 \leqq t \leqq 1$.

The function $\sigma$ will be so chosen that $\sigma(0)=0$, whereby the interval of values for $\sigma$ becomes $(0, \lambda)$, if $\lambda=\sigma(1)$; but this remark is unimportant, to the extent that the possibility of making such a choice of $\sigma$ is clearly implied in the theorem as already stated, since the subtraction of a constant from $\sigma(t)$ does not affect any property of it with which the theorem is concerned; and it would be possible further to replace the value $\lambda$ by 1 .

As a consequence of the theorem, it is to be observed that within the limits $0 \leqq t \leqq 1,0 \leqq \sigma \leqq \lambda$, to every value of $t$ corresponds one (and just one) value of $\sigma$, and to every value of $\sigma$ corresponds at least one value of $t$, so that every point $(x, y)$ given by the relations (1) is given by the equations

$$
x=F(\sigma), \quad y=\Phi(\sigma),
$$

and vice versa. But it is not true in general that $t$ is a singlevalued function of $\sigma$.

To prove the theorem, let us notice first that it is practically obvious if the curve (1) is rectifiable, the arc-length $s$, defined in the usual way as limit of inscribed polygons, serving the purpose of $\sigma$. The function $s(t)$ is certainly single-valued, continuous, and non-decreasing. * To every value of $s$ between 0 and $l$, where $l$ is the total length of the curve, corresponds at least one value of $t$, in consequence of the properties just named, and so at least one point $(x, y)$ of the curve. Furthermore, although $t$ need not be a single-valued function of $s$, a specified value of $s$ can not give more than one point $(x, y)$, since the values of $s$ for any two distinct points differ by at least the length of the chord joining the points. So $x$ and $y$ are single-valued functions of $s$, which may be denoted by $F(s)$ and $\Phi(s)$, defined for $0 \leqq s \leqq l$, and fulfilling the identities $F(s)=f(t), \Phi(s)=\varphi(t)$, from the manner of their p. 585 .

* Cf., e. g., Pierpont, Theory of Functions of Real Variables, vol. 2, 
definition. They are continuous, since $\Delta x \leqq \Delta s, \Delta y \leqq \Delta s$; and they are evidently not constant together over any interval. A proof of the last statement, labored as the proof of an evident fact is likely to be, is as follows: Because of the monotone character of $s$, an interval of values of $s$ corresponds to an interval of values of $t$. If $F$ and $\Phi$ were constant throughout an $s$-interval, $f$ and $\varphi$ would be constant throughout the corresponding $t$-interval; but then this $t$-interval would give just a single point $(x, y)$, and so a single value, not an interval of values, of $s$. So the theorem is established for any rectifiable curve.

Now consider an arbitrary continuous curve, given in the form (1). Let $\omega(\delta)$ be the maximum of

$$
\sqrt{\left[f\left(t^{\prime \prime}\right)-f\left(t^{\prime}\right)\right]^{2}+\left[\varphi\left(t^{\prime \prime}\right)-\varphi\left(t^{\prime}\right)\right]^{2}}
$$

for $0 \leqq t^{\prime} \leqq 1,0 \leqq t^{\prime \prime} \leqq 1,\left|t^{\prime}-t^{\prime \prime}\right| \leqq \delta$. This function is continuous and non-decreasing for all values of $\delta \geqq 0$. The latter property is evident; the former follows from the facts that

$$
\lim _{\delta=0} \omega(\delta)=0, \quad \omega\left(\delta_{1}+\delta_{2}\right) \leqq \omega\left(\delta_{1}\right)+\omega\left(\delta_{2}\right) .
$$

The function $\omega(\delta)+\delta$ is continuous and increasing. Let $\psi$ be the inverse function, so that

$$
\delta=\psi[\omega(\delta)+\delta]
$$

then $\psi$ also is single-valued, continuous, and increasing, and $\psi(0)=0$.

Let $t$ be any point in the interyal $(0,1)$. Let $t_{1}, t_{2}, \cdots, t_{n-1}$ be any $n-1$ points between 0 and $t$, arranged in order, and let $t_{0}=0, t_{n}=t$. Let

$\delta_{i}=\sqrt{\left[f\left(t_{i}\right)-f\left(t_{i-1}\right)\right]^{2}+\left[\varphi\left(t_{i}\right)-\varphi\left(t_{i-1}\right)\right]^{2}} \quad(i=1,2, \cdots, n)$, and, finally, let

$$
\sigma(t)=\text { upper limit of } \sum_{i=1}^{n} \psi\left(\delta_{i}\right)
$$

for all possible choices of $t_{1}, \cdots, t_{n-1}$ and all values of $n$. This function $\sigma(t)$ will be shown to fulfil the requirements of the theorem.

In the first place, $\sigma(t)$ is defined for $0 \leqq t \leqq 1$. For

$$
\delta_{i} \leqq \omega\left(t_{i}-t_{i-1}\right)<\omega\left(t_{i}-t_{i-1}\right)+\left(t_{i}-t_{i-1}\right),
$$


and consequently

and

$$
\psi\left(\delta_{i}\right)<t_{i}-t_{i-1}
$$

$$
\sum_{i=1}^{n} \psi\left(\delta_{i}\right)<t
$$

so that the upper limit in (2) is finite. It is evident that $\sigma(t)$ can not diminish when $t$ increases.

Let $\sigma\left(t^{\prime}, t^{\prime \prime}\right)$ denote the upper limit of the sums $\Sigma \psi\left(\delta_{i}\right)$ obtained by subdividing the interval $\left(t^{\prime}, t^{\prime \prime}\right)$; in particular, $\sigma(0, t)=\sigma(t)$. The functions $\sigma$ with one and two arguments respectively will not be in any danger of confusion. The proof that $\sigma(t)$ is continuous resembles the corresponding proof for rectifiable curves, ${ }^{*}$ but it is necessary to exercise some care, for two reasons. In the first place, a sum $\Sigma \psi\left(\delta_{i}\right)$ may be diminished by introducing new points of subdivision and retaining the old ones; it may even happen that the greatest possible value of the sum is attained by taking $n=1$, and in no other way. $\dagger$ Secondly, if $t^{\prime}<t^{\prime \prime}<t^{\prime \prime \prime}$, while it is evident that

$$
\sigma\left(t^{\prime}, t^{\prime \prime \prime}\right) \geqq \sigma\left(t^{\prime}, t^{\prime \prime}\right)+\sigma\left(t^{\prime \prime}, t^{\prime \prime \prime}\right),
$$

the sign of equality need not hold. $\ddagger$

Let $c$ be any point in the interval $0<c \leqq 1$. It will be shown first that $\sigma(t)$ is continuous on the left at the point $c$. Since $\sigma$ is monotone increasing, it is required only to show that if $\epsilon$ is any positive quantity, there is a $t<c$ such that

$$
\sigma(t)>\sigma(c)-\epsilon .
$$

Take any subdivision of $(0, c)$ such that the corresponding sum $\Sigma \psi\left(\delta_{i}\right)$ is greater than $\sigma(c)-\frac{1}{2} \epsilon$. For $t>t_{n-1}$, let

$$
\delta_{n}{ }^{\prime}=\sqrt{\left[f(t)-f\left(t_{n-1}\right)\right]^{2}+\left[\varphi(t)-\varphi\left(t_{n-1}\right)\right]^{2}} .
$$

If $t$ is sufficiently near to $c, \delta_{n}{ }^{\prime}$ will differ so little from $\delta_{n}$ that $\$$ $\psi\left(\delta_{n}{ }^{\prime}\right)>\psi\left(\delta_{n}\right)-\frac{1}{2} \epsilon$, and the points $t_{1}, \cdots, t_{n-1}$ give a subdivision of $(0, t)$ such that the corresponding sum $\Sigma \psi\left(\delta_{i}\right)$ exceeds $\sigma(c)-\epsilon$. Hence (3) is satisfied.

In the same way, it may be shown that, for $0 \leqq c<1$,

* Cf., e. g., Lebesgue, Leçons sur l'Intégration, pp. 53-54, 60.

$\dagger$ E. g., let $f(t)=\sqrt{t}-t, \varphi(t)=0$. Then $\omega(\delta)=\sqrt{\delta}-\delta, \psi(\delta)=\delta^{2}$, and $\sigma(1)=1=\psi(1)$, while $\Sigma \psi\left(\delta_{i}\right)<1$ if $n>1$.

$\ddagger$ In the example of the preceding footnote, $\sigma\left(0, \frac{1}{2}\right)+\sigma\left(\frac{1}{2}, 1\right)=\frac{1}{2}$.

$\S$ Of course it is not inconceivable that $\psi\left(\delta_{n}{ }^{\prime}\right)>\psi\left(\delta_{n}\right)$. 


$$
\sigma(t, 1)>\sigma(c, 1)-\epsilon,
$$

if $t$ is greater than $c$ and sufficiently near to $c$. Since

it follows that

$$
\sigma(c, 1) \geqq \sigma(c, t)+\sigma(t, 1),
$$

$$
\sigma(c, t)<\epsilon ;
$$

but even now it can not be inferred immediately (except for $c=0)$ that $\sigma(t)$ is continuous on the right at $c$, because of the failure of the additive property for consecutive intervals.

Let $\epsilon$ be any positive quantity. Let $t>c$ be so close to $c$ that $\sigma(c, t)<\frac{1}{3} \epsilon$, which is possible, in consequence of the preceding paragraph; and so close also that the length of the chord joining the points $[f(c), \varphi(c)]$ and $\left[f\left(t^{\prime}\right), \varphi\left(t^{\prime}\right)\right]$ is less than $\frac{1}{3} \epsilon$ for $c \leqq t^{\prime} \leqq t$. If $\delta^{\prime}$ and $\delta^{\prime \prime}$ are the lengths of the chords joining these two points with any third point, then $\left|\delta^{\prime \prime}-\delta^{\prime}\right|<\frac{1}{3} \epsilon$; and it follows that

$$
\left|\psi\left(\delta^{\prime \prime}\right)-\psi\left(\delta^{\prime}\right)\right|<\frac{1}{3} \epsilon,
$$

since any increment of $\omega(\delta)+\delta$ is greater than or equal to the corresponding increment of $\delta$, because of the monotone character of $\omega$, and any increment of $\psi$ consequently is less than or equal to the corresponding increment of its argument.

After choice of a particular value of $t$ satisfying the conditions just specified, take any subdivision of the interval $(0, t)$ such that the corresponding sum $\Sigma \psi\left(\delta_{i}\right)$ exceeds $\sigma(t)-\frac{1}{3} \epsilon$, the points of division being $t_{0}=0, t_{1}, \cdots, t_{n-1}, t_{n}=t$. Suppose $k$ is the index such that $t_{k-1}<c \leqq t_{k}$. Then the terms $\psi\left(\delta_{k+1}\right)+\cdots+\psi\left(\delta_{n}\right)$ constitute a sum which can not exceed $\sigma(c, t)$, and so is less than $\frac{1}{3} \epsilon$. If $\delta_{k}{ }^{\prime}$ is the length of the chord joining $\left[f\left(t_{k-1}\right), \varphi\left(t_{k-1}\right)\right]$ and $[f(c), \varphi(c)]$, then

$$
\psi\left(\delta_{k}\right)-\psi\left(\delta_{k}{ }^{\prime}\right)<\frac{1}{3} \epsilon,
$$

in consequence of (4). Hence

$\psi\left(\delta_{1}\right)+\cdots+\psi\left(\delta_{k-1}\right)+\psi\left(\delta_{k}{ }^{\prime}\right)>\psi\left(\delta_{1}\right)+\cdots$

and it follows that

$$
+\psi\left(\delta_{n}\right)-\frac{2}{3} \epsilon>\sigma(t)-\epsilon,
$$

$$
\sigma(c)>\sigma(t)-\epsilon,
$$

which proves the continuity of $\sigma(t)$ on the right at $c$. 
The rest of the proof of the theorem is almost immediate. In consequence of the continuity of $\sigma(t)$ there corresponds to every value of $\sigma$ between 0 and $\lambda=\sigma(1)$ at least one value of $t$, and so at least one point of the curve. There can not be more than one point for a single value of $\sigma$, because the values of $\sigma$ corresponding to any two distinct points differ by at least $\psi(\delta)$, if $\delta$ is the length of the chord joining the points. If we set $x=F(\sigma), y=\Phi(\sigma)$, these are single-valued functions of $\sigma$, and are identically equal to $f(t)$ and $\varphi(t)$ respectively, by their very definition. They are continuous, because

$$
\Delta \sigma \geqq \psi\left(\sqrt{\Delta x^{2}+\Delta y^{2}}\right), \quad \Delta x \leqq \sqrt{\Delta x^{2}+\Delta y^{2}} \leqq \omega(\Delta \sigma)+\Delta \sigma,
$$

and similarly for $\Delta y$. Finally they are not constant together in any interval, by the same argument as in the case of a rectifiable curve.

The extension of the work to curves in three or more dimensions requires only the writing down of a correspondingly larger number of symbols.

HaRvard UNIVERSity, Cambridge, Mass.

\section{JOHN WALLIS AS A CRYPTOGRAPHER.}

BY PROFESSOR DAVID EUGENE SMITH.

IT is not probable that many bibliophiles in the domain of mathematics, seeing upon their shelves the sumptuous tall copies of the Opera Mathematica* of John Wallis, and consulting their noteworthy historical chapters, the first serious effort in the history of mathematics in England, ever consider that the author was one of the world's greatest decipherers of cryptic writing. To be sure his biographies give us the information that he was interested in cryptography, but the extent of this interest, the sixty years devoted to the subject, the services rendered to the State, the rewards and disappointments that came to him as a result-of all this the biographies tell us practically nothing. It is partly because of this fact,

* Oxoniæ, MDCXCV. 